\title{
SUPER-LIGHT MOTION IN RADIO SOURCES AND ITS IMPLICATIONS FOR THE DISTANCE SCALE PROBLEM
}

\author{
K. I. KELLERMANN and D. B. SHAFFER \\ National Radio Astronomy Observatory
}

Plusieurs radio sources comme $3 \mathrm{C} 345$ et $3 \mathrm{C} 120$ sont constituées de plusieurs composantes qui semblent se séparer avec une vitesse apparente de 5 ou 10 fois la vitesse de la lumière. Ce phénomène peut être interprété comme une preuve contre l'interprétation cosmologique du décalage vers le rouge. D'autres explications tirées de la physique et de la cosmologie conventionnelles sont abordées mais elles ne sont pas tout à fait satisfaisantes.

Since 1971 there has been growing evidence that in some extragalactic radio sources there are two or more components which appear to separate with a high angular velocity. If these sources are located at distances corresponding to their redshift, then the apparent component velocities are considerably greater than the speed of light, and this has been taken as evidence against the cosmological origin of quasar redshifts.

The first evidence for apparent super-light motions came from observations of rapid flux density variations observed in several quasars. Assuming that the linear diameter $d \lesssim c \tau$, where $\tau$ is the characteristic time scale of the variations, an upper limit to the angular size, $\theta$, is given by $\theta \lesssim H \tau / z$. The corresponding brightness temperature in some cases apparently exceeded by several orders of magnitude the limit of $10^{11-12} \mathrm{~K}$ for an incoherent synchrotron source (Kel lermann and Pauliny-Toth 1969), and the apparent energy supply over periods as short as a few months was in excess of $10^{60}$ ergs* (Pauliny-Toth and Kellermann 1966). These problems are removed if the quasars are closer than indicated by their redshift.

Rees (1967), however, has argued that if a source expands with a highly relativistic velocity, then the difference in arrival time of the

*H $=50 \mathrm{~km} \mathrm{sec}^{-1} \mathrm{Mpc}^{-1}$ and $\mathrm{q}_{0}=0.05$ are assumed throughout this paper. 
signai emitted from different parts of the source can cause the appearance of a source expanding with an apparent transverse velocity $V \sim \gamma_{c}$, so that the apparent diameter may exceed that given by the light travel time by a factor $\gamma{ }^{*}$ Values of $\gamma$ in the range 3 to 10 ( $\beta$ in the range 0.95 to 0.99 ) are sufficient to reduce the energy requirements (approximately proportiona1 to $\gamma^{-2}$ ) and inverse Compton scattering (proportional to $\gamma^{-9}$ ) to acceptable values, although the presence of surprisingly rapid variations observed at relatively long wavelengths in some quasars are still difficult to explain in this way (Hunstead 1972, Jones and Burbidge 1973).

If this relativistic expansion model is correct or if the quasars are not located at cosmological distances, then it was expected that an increase in the angular size should be observable with very-long-baseline interferometer (VLBI) techniques. Thus the subsequent direct measurement of apparent super-light motions in some quasars did not come as a complete surprise. However, the actual situation is more complex than expected from the simple Rees model. The typical source is not a simple expanding cloud, but appears to contain two or more components which appear to separate with velocities 5 to 10 times the speed of light. Moreover, because of the complex geometry and the limited number of interferometer baselines which have been used, the interferometer observations do not uniquely determine the radio structure, and there has been some ambiguity in the evidence for super-1ight motion.

The first interferometer observations of apparent super-1ight motion came from a series of transpacific observations made between 1967 and 1969. These data indicated that the size of the variable components in $3 \mathrm{C} 273$ and 3C 279 were $\gtrsim 25$ light years in diameter only 3 years after the expansion began, so that the apparent expansion velocity was $\gtrsim 4 c$ (Gubbay et al. 1969, Moffet et a1. 1972). However, this result was based on only a single interferometer spacing assuming a circular gaussian geometry, and that the expansion began when the flux density suddenly increased in 1966. As it turns out, although all of these assumptions were probably incorrect, the more recent detailed interferometer data still indicate super-light motion in these sources.

More direct evidence for apparent faster-than-light-motion came in

$* \gamma=\left(1-\beta^{2}\right)^{-\frac{1}{2}}, \beta=v / c, v=$ expansion velocity in the rest frame of the source. 
1971. In October 1970, observations made with a transcontinental tracking interferometer showed that 3C 273 and 3C 279 had a surprisingly complex structure unlike what might have been expected from the simple expanding cloud model (Knight et al. 1971). A satisfactory fit to the data could be obtained with two-component models. However, as is always the case with interpreting interferometer data from a single baseline, a more complex geometry could not be excluded. These observations were repeated in February 1971 (Cohen et a1. 1971, Whitney et al. 1971), and it was discovered that the structure had changed remarkably in only a few months. Based on the double model, the two components in both sources, but particularly $3 \mathrm{C} 279$, appeared to have separated with a linear velocity of 5 to 10 times the speed of light. It was also clear, however, that with a more complex geometry there was no requirement for actual motion (Cohen et al. 1971, Whitney et a1. 1971, Dent 1972). Specifical1y, a fixed three-component model, in which the central component varied with time, could fit all of the data without any real motion. However, preliminary data then available indicated that at least for 3C 279 the total flux density had not changed (Dent 1971), so a rather contrived balance of the various components was required (Cohen et a1. 1971). Later, Dent (1972) found evidence for a small flux density change and argued for the fixed-component-variable-flux model. The single-baseline data available did not allow the distinction between these two classes of models.

In order to distinguish between the expanding double and fixed multiple component models, repeated observations using several simultaneous interferometer pairs have been made at several wavelengths over the past five years. The new data come primarily from the NRAO-Caltech-Bonn group and the MIT-NASA group, which have used stations in Europe and the USA to obtain multi-element data which allow the source brightness distributions to be determined in some detail. The current situation for several of the well-observed sources is summarized below.

3C 273

The first three-baseline observations made in April 1972 at $2.8 \mathrm{~cm}$ wavelengths showed that the structure of 3C 273 could not in fact be described by a simple double model (Shaffer 1973). A simple geometry of 3 linear components did give a good fit to the data, and it was shown that variations in the intensity of these components could reproduce all of the 
data for 1971 without the need for any real motion (Kellermann et a1. 1974). However, continuation of the multi-element interferometry indicated that the three components in fact appeared to be separating, and that at least one component was increasing in size (Schilizzi et al. 1975, Kellermann et a1. 1977). On the other hand, Legg et a1. (1976) were able to fit all the data through 1973 with a fixed-component, variable-flux geometry. Their models fit the data without any need for component motion, but at least one component itself appears to expand with apparent super-light velocity.

The complex geometry and low declination of $3 \mathrm{C} 273$ do not permit a unique distinction between models which have parts separating with apparent super-light velocity, or ones where parts of the source successively fade and brighten with time; but observations by several independent groups over a range of wavelengths do show that the apparent size has steadily increased since late 1970 with a mean apparent velocity of $\sim 5 c$. Extrapolation of the component separation to zero spacing indicates that the separation began in 1966, close to the time of a large increase in flux density which was observed at $\mathrm{cm}$ wavelengths (e.g. Kellermann and Pauliny-Toth 1969).

\section{C 345}

More clear-cut evidence of apparent super-light separation has been presented by Cohen et a1. (1976) and Wittels et a1. (1976) in the double source 3C 345. Cohen et a 1 . observed at $2.8 \mathrm{~cm}$ during the period 1974 to 1976 and quoted an apparent velocity of 8c. Wittels et al. presented data taken at $3.8 \mathrm{~cm}$ between 1972 and 1974 and quoted a velocity of $\sim 3 \mathrm{c}$. Although the apparent velocities measured by the two groups are somewhat discrepant, inspection of all the data, which are shown in Figure 1, shows that in 1974 when both groups had data, the measured separation at 2.8 and $3.8 \mathrm{~cm}$ agreed very we11, and that the rate of change in apparent separation appears to have increased between 1971 and 1975.

Figure 1 also gives other unpublished data at $2.8 \mathrm{~cm}$ as well as at $2 \mathrm{~cm}$ (Shaffer et a1. 1977). The data at the different wavelengths are in good agreement and are consistent with a double source lying along position angle $\sim 105^{\circ}$. The separation has increased with an apparent velocity increasing from $\sim 3 c$ prior to 1974 to $\sim 8 c$ in 1974 and 1975 . The most recent data indicate a possible abrupt cessation of the expansion in 1976, but more data will be required to confirm this. 


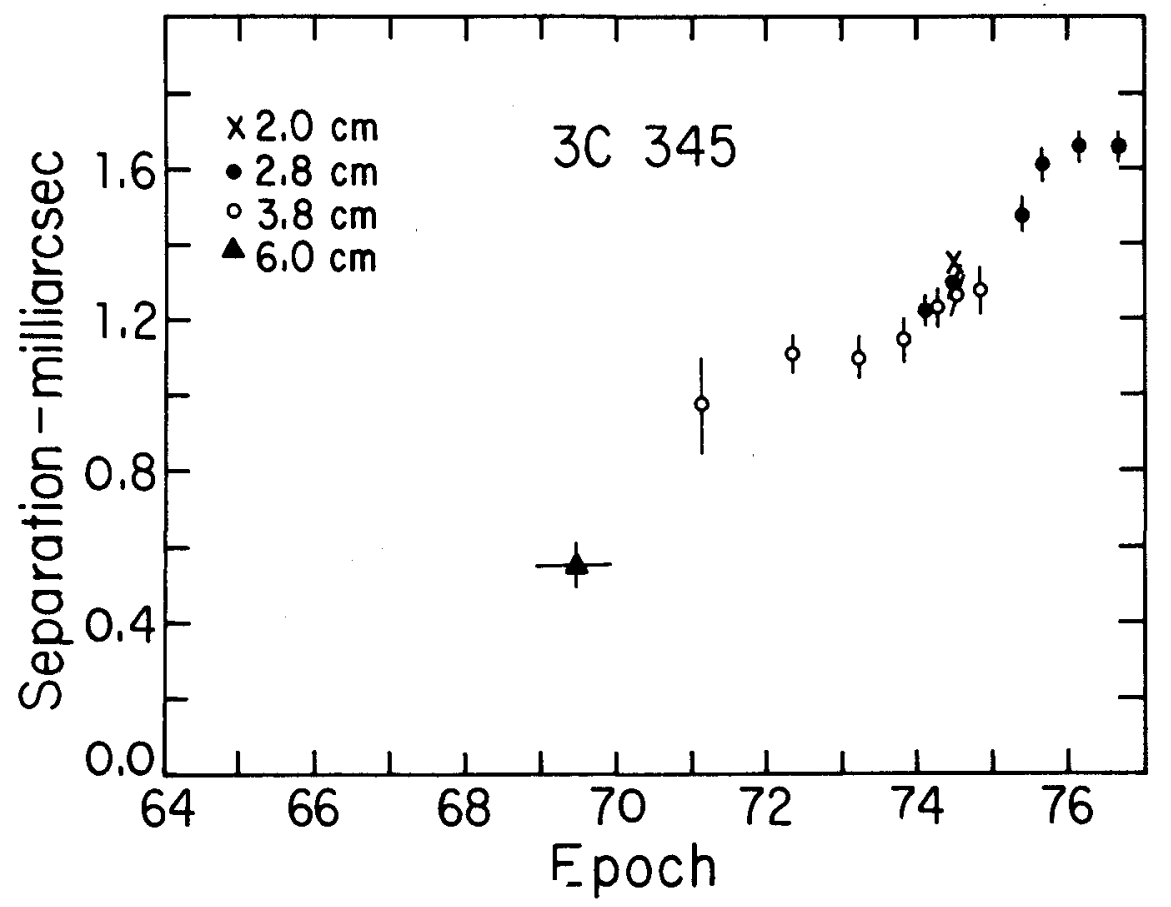

Fig. 1. - Apparent separation of the two components of $3 \mathrm{C} 345$ as a function of time. The point at $6 \mathrm{~cm}$ was taken from a number of measurements made over a period of about 1 year. The source was only slightly resolved, and the separation shown for 1969 is based on an assumed double source model oriented in position angle $105^{\circ}$.

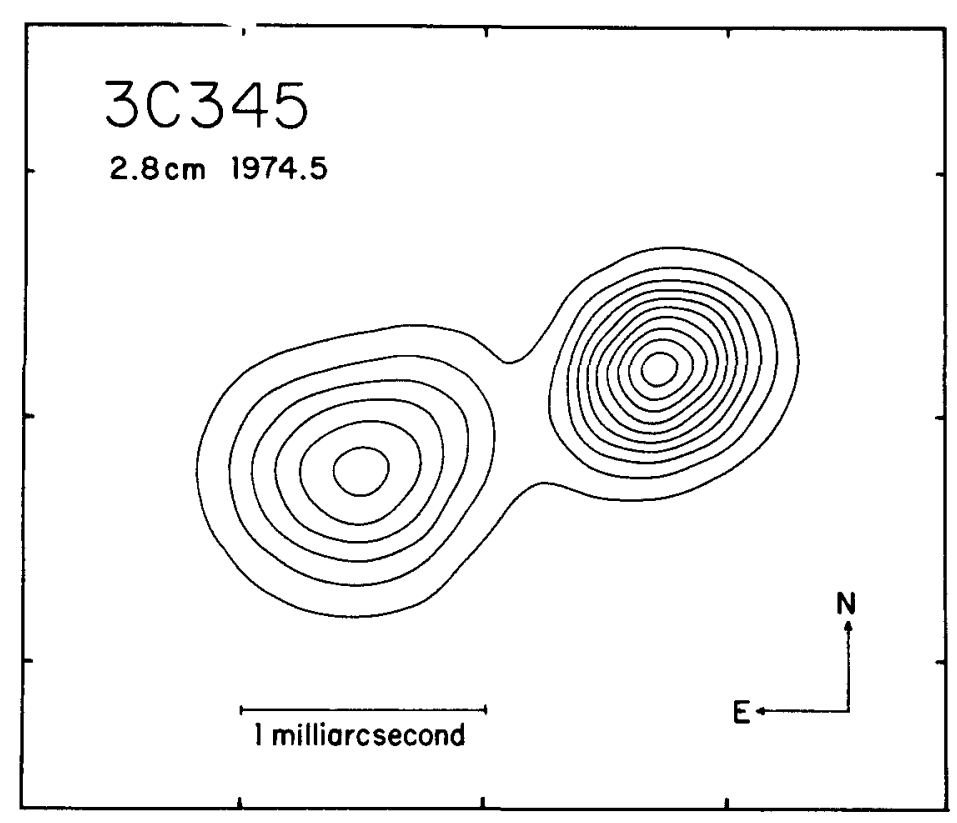

Fig. 2. - The observed brightness distribution of $3 \mathrm{C} 345$ at $2.8 \mathrm{~cm}$ at the epoch 1974.5 (Shaffer et al. 1977). 
The data for 3C 345 show a well-defined double source (Figure 2), with no evidence for any third component (Wittels et a1. 1976, Shaffer et al. 1977). Thus, considering that the total flux density has changed only slightly since 1971, while the separation has increased from 1.1 to 1.8 milli-arcsec, it does not seem possible to interpret the apparent superlight separation of $3 \mathrm{C} 345$ with a fixed-component variable-flux model.

Extrapolation of the apparent velocity to zero separation indicates that an "event" occurred between 1964 and 1968 depending on whether the velocity is assumed constant or was accelerating. Figure 3 shows the flux density observed for $3 \mathrm{C} 345$ at $2 \mathrm{~cm}$. A large increase in flux density was observed in 1966 (Kellermann and Pauliny-Toth 1969), near the time of the start of the apparent expansion.

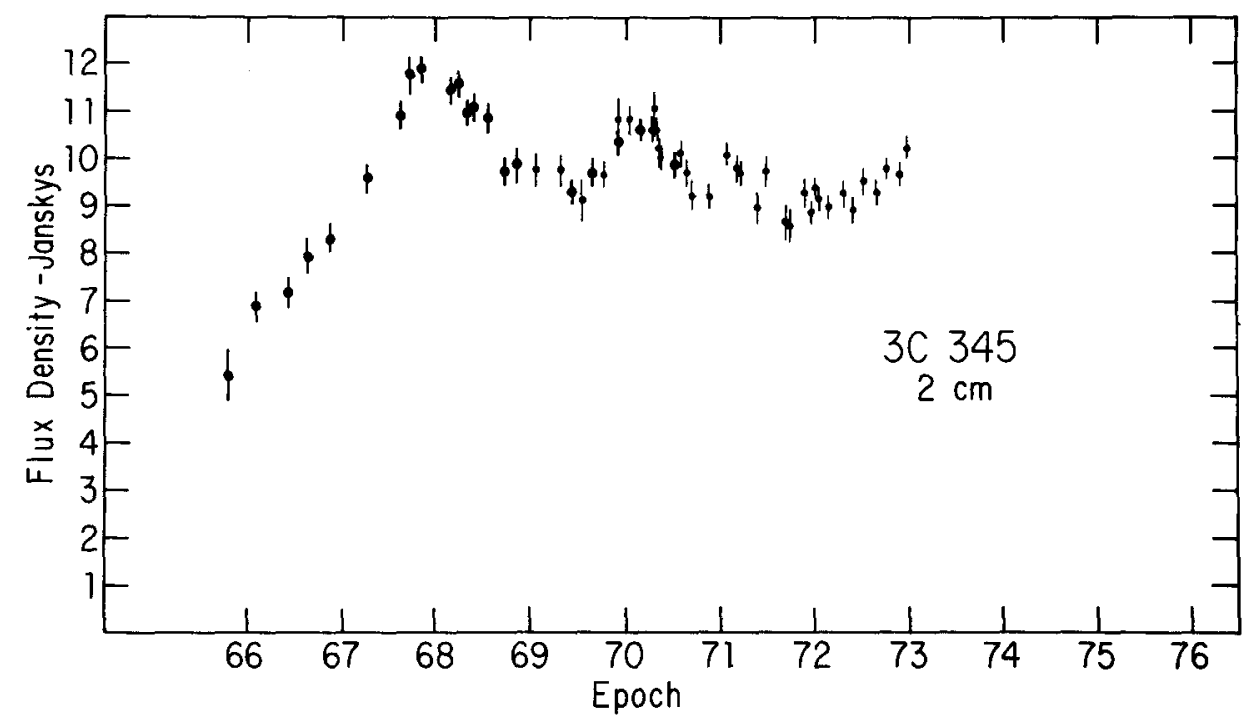

Fig. 3.- Flux density of $3 C 345$ observed at $2 \mathrm{~cm}$. The large circles are taken from Kellermann and Pauliny-Toth (1968 and unpublished), and the smal1 circles from Dent et al. (1974).

3C 120

A much more complex situation exists in the radio source $3 C$ 120, which is identified with a Seyfert-type galaxy which has had numerous radio outbursts. Seielstad (1974) has shown the observed variation in total flux is consistent with the model of Ozernoi and Sazonov (1969) which postulates successive outbursts forming two relativistically separating and expanding clouds of relativistic particles. The long baseline interferometer observations, which go back to 1971, give more direct evidence of a separating double source. All of the observations can be interpreted in terms of double source models. 


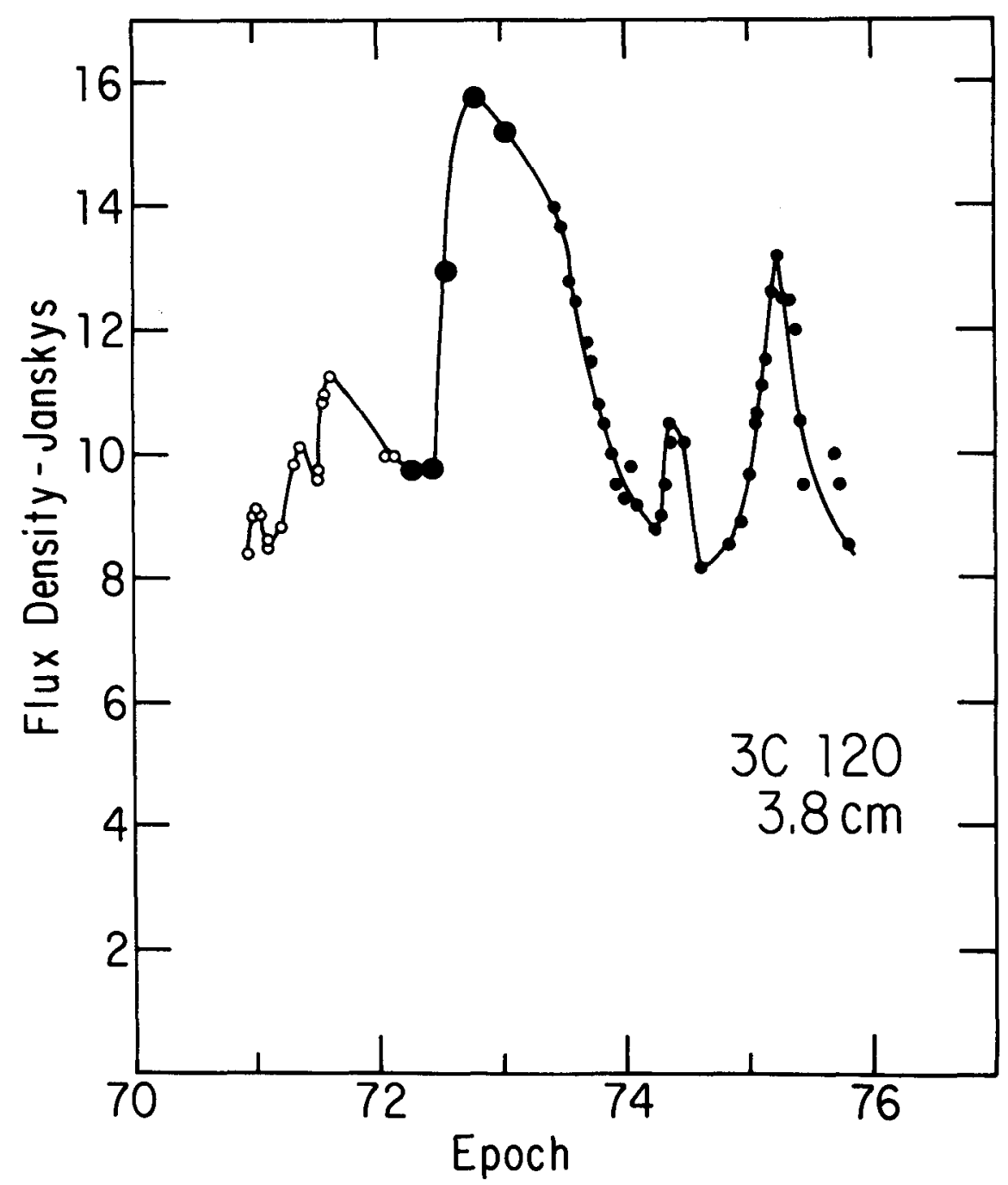

Fig. 4.- Variations in flux density of 3C 120 at $3.8 \mathrm{~cm}$. Open circles are from Dent and Kojoian (1972), large closed circles from Kellermann et al. (1973), and the small closed circles from Williams and BTankenship (private communication).

Figure 4 and 5 show the observed variation in total flux density at $3.8 \mathrm{~cm}$ and the apparent separation of the components as observed at $2.8 \mathrm{~cm}$, $3.8 \mathrm{~cm}$, and $6 \mathrm{~cm}$. The limited data taken in 1971 at $3.8 \mathrm{~cm}$ have already been published and have been interpreted as evidence for component separation with a velocity $\sim 2.5 \mathrm{c}$ (Shaffer et a1. 1972). Later measurements made in 1972 indicated another weak outburst, followed by a much larger outburst which reached a peak near the end of 1972 (Kellermann et a1. 1973, Shapiro et al. 1973) and decayed until early 1974. Figure 5 shows that at first this outburst was unresolved $(\theta \lesssim 0.3 \mathrm{milli- \operatorname {arcsec }})$, but that by late 


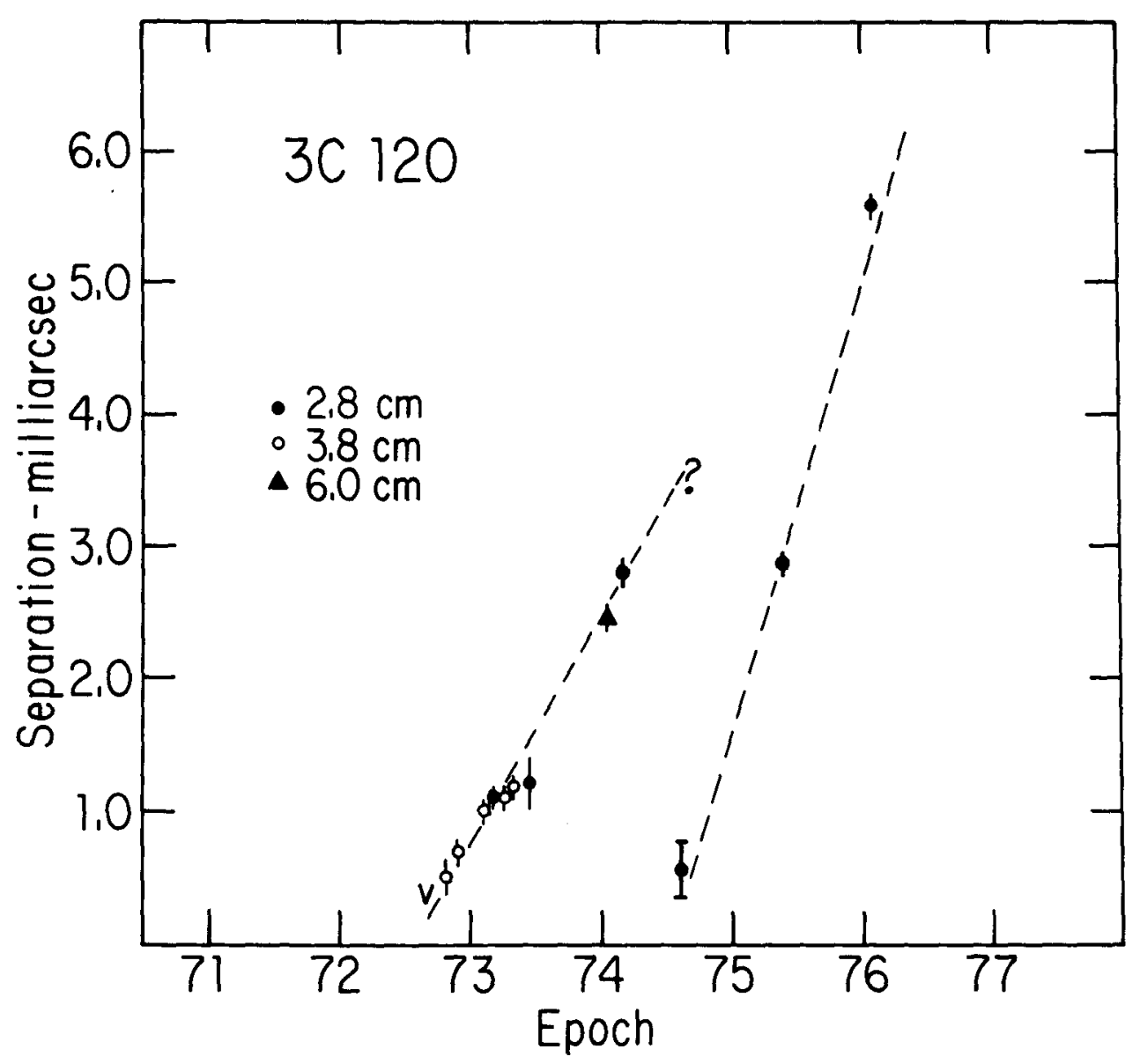

Fig. 5. - Variation in separation of the components of $3 \mathrm{C} 120$ at several wavelengths as a function of time.

1972 it was just resolved, and after 1973.2 it was clearly resolved into a double source. The component separation then appeared to increase at a rate of 1.6 milli-arcsec per year (3.5c) until the outburst died out in 1974. At about that time a new outburst began, and the component separation was determined to be only $\sim 0.5 \mathrm{milli}$-arcsec in mid-1974. The separation increased during 1975 at the surprisingly rapid rate of $3.2 \mathrm{mil1i-}$ arcsec per year (7c).

During both of these outbursts the position angle of separation remained constant near 65 degrees, and the data at different wavelengths are in essential agreement. In both cases the apparent separation of the components increased by more than a factor of 10 on a time scale of the order of one year, and at least for the last observation there was no 
evidence of any third component containing more than a few percent of the total flux density. It does not seem possible therefore to explain the apparent rapid separation of the components in terms of any fixed-component variable-flux model.

\section{C 39.25}

Not all compact sources show a change in separation with time. In the quasar 4C 39.25 (see Figure 6), the separation ( 2.0 milli-arcsec) and position angle have remained essentiatly constant over a two-year period, while the component fluxes have slowly changed (Shaffer et al. 1977). The observations show no evidence for an increase in separation at a rate greater than 20 or 30 micro-arcsec per year, corresponding to an upper

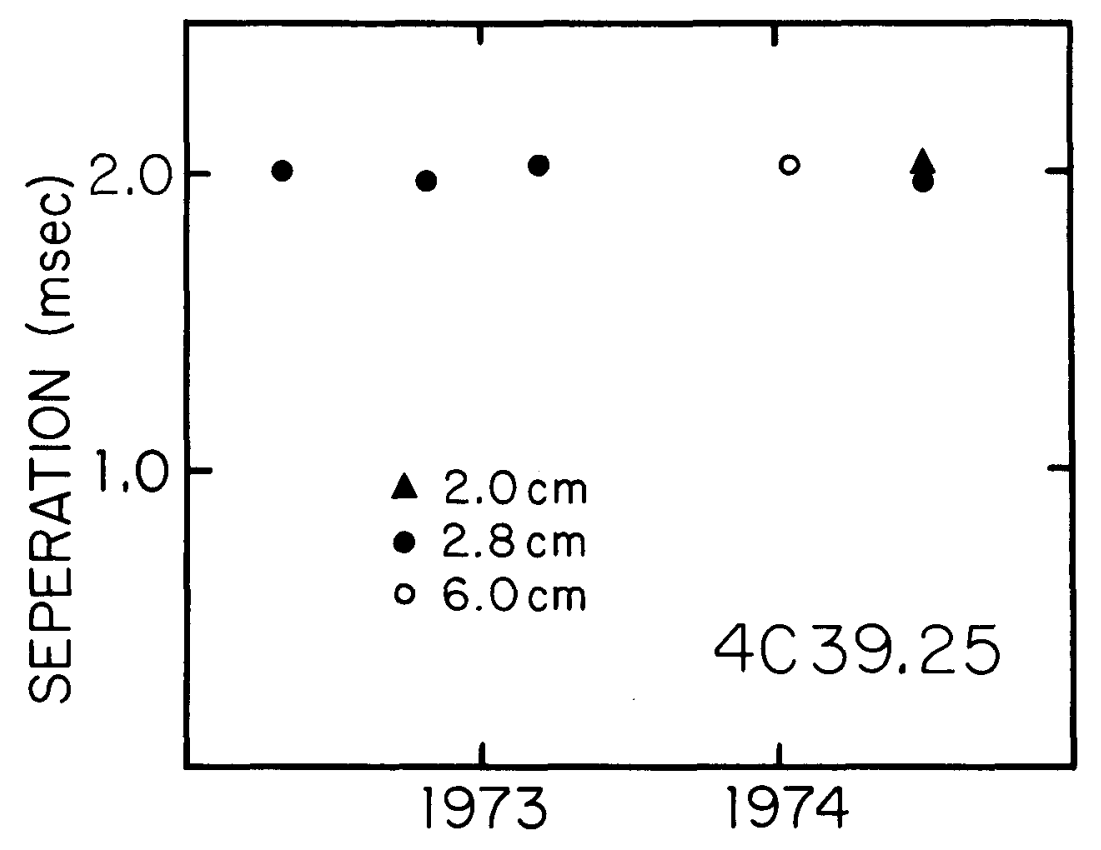

Fig. 6. - Separation of the components of $4 C 39.25$ as a function of time.

limit to the apparent linear velocity of $\sim c$. This is a clear example of a fixed-component variable-flux source. Similarly, the radio source in the nucleus of NGC 1275 (Pauliny-Toth et a1. 1976) and in BL Lac (Kellermann et al. 1977) have multiple-component structure where parts of the source brighten and fade, and where there is no evidence of any motion. 
Summary

Compact radio sources generally have a multi-component structure which changes with time. The observed variations range from a simple change in flux density of one or more fixed components (e.g. 4C 39.25) to a separation of two components with an apparent velocity greater than the

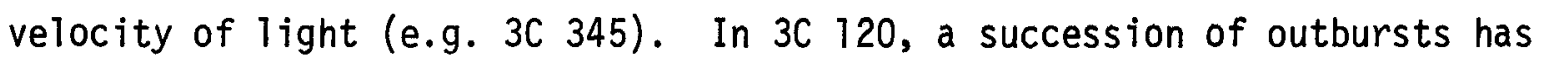
occurred, each producing a pair of rapidly separating components. In $3 \mathrm{C} 273$ the situation is ambiguous, due to the complexity of the source structure and the low declination of the source.

The form of the apparent super-light motion may be summarized as follows:

a) The observed component separation at any epoch is independent of wavelength, so that interpretations in terms of a refractive effect or variations in opacity (e.g. Epstein and Geller 1976) are unlikely.

b) When sufficient data are available to show a trend, it is always an expansion, never a contraction. Occasionally, a source is suddenly smaller than the previous measurements, but this is associated with an intensity change, suggesting the appearance of a new pair of expanding components.

c) The position angle determined by the components remains essentialiy constant as the components separate. Where successive pairs of separating components are observed, they all lie along the same position angle.

d) The apparent velocity of separation is not always constant. Apparent accelerations and decelerations are observed, and the individual measurements of component separation deviate significantly from a smooth curve.

e) Changes in the component separation of up to a factor of 10 are observed, with apparent linear velocities of up to $10 \mathrm{c}$.

f) The epoch of zero component separation determined by extrapolation of the observed data often lies close to the start of a major outburst in flux density.

At least for $3 C 345$ and for the most recent outburst in $3 C$ 120, the data strongly suggest simple double-source models, and we cannot interpret the large change in separation in these sources in terms of a fixed-component variable-flux model. 
It is tempting to speculate on the existence of real super-light particles (i.e. tachyons) in quasars and galactic nuclei where extreme physical conditions may exist. However, a variety of less exotic, if perhaps somewhat contrived, explanations have

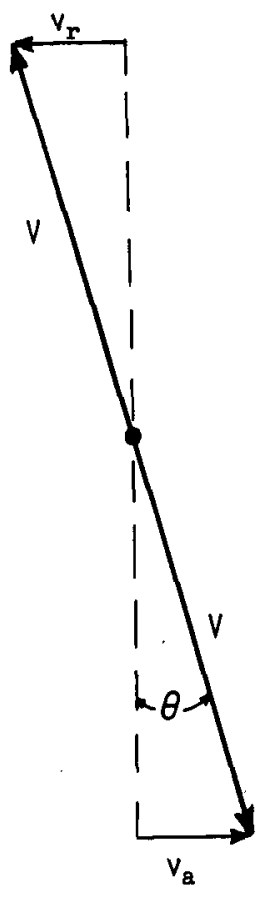

Fig. 7.- Geometry giving rise to apparent faster-than-1ight motion due to finite signal propogation time. been offered in terms of various illusions or phase velocity effects. One of the most attractive interpretations is that the apparent super-light motion is due to the differential light travel time of a signal emitted from an object which is approaching the observer at relativistic velocities. This is a variant of the Rees (1967) relativistic expansion model.

If we suppose that a source of synchrotron emission is approaching the observer with a highly relativistic velocity, $v$, and makes an angle $\theta$ with the line of sight (Figure 7 ), the apparent transverse linear velocity, $v_{a}$, is then

$$
v_{a}=v \sin \theta /(1-\beta \cos \theta) \text {, where } \beta=v / c \text {. }
$$

This is maximized when

$$
\sin \theta=1 / \gamma \text {, where } \gamma=\left(1-\beta^{2}\right)^{-\frac{1}{2}} \text {, }
$$

and then

$$
v_{a}(\max ) \sim \gamma c \text { for } \gamma>1
$$

For a receding component, $V_{r} \sim c / 2 \gamma$.

These results are only slightly modified if one maximizes the relative velocity of two components moving in opposite direction in the source rest frame, rather than that of the approaching component. In this case

$$
V=2 v \sin \theta /\left(1-\beta^{2} \cos ^{2} \theta\right), \sin \theta_{\max }=1 / \beta \gamma, V_{(\max )}=\gamma c \text {. }
$$


Thus if $\theta$ is sma11, the receding component appears to move relatively slowly compared with the approaching component. With a typical value $V_{\max } \sim 5 c$, $\gamma \sim 5, \theta \sim 10^{\circ}$, and $v \sim .98 \mathrm{c}$. These models thus require bulk motions with velocities close to the velocity of light. A typical synchrotron source has relativistic particles with $\gamma \sim 1000$, so that collective motion with $\gamma \sim 5$ or 10 is perhaps not unexpected. The relativistic motion model is also attractive in that it provides a natural explanation of the observed rapid intensity variations as simply the effect of relativistic time dilation.

There are, however, difficulties with this model. It has been pointed out that the luminosity of an approaching component will be modified by the Doppler shift in the ratio (e.g. Cohen et al. 1971)

$$
S_{a} / S_{0}=(1+\beta \cos \theta)^{3-\alpha}
$$

where $\alpha$ is the spectral index defined by $S_{0} \propto \nu^{\alpha}$. For a value of $\beta \sim 1$, $\cos \theta \sim 1$, and $\alpha \sim 0, S_{a} / S_{0} \sim 10$. The receding component $(\cos \theta \sim-1)$ is essentially invisible $\left(S_{r} / S_{a} \sim 10^{-6}\right)$, but the observed component ratios are typically less than 1.5 to 1 . As discussed above, the receding component does not contribute significantly to the apparent rapid velocity, so that possibly we are seeing the relative motion of an approaching component and one which is at rest, but still the absence of the expected intensity ratio of $\sim 10$ to 1 is difficult to explain. Alternately, there may be many components which are ejected more or less isotropically from a common origin. Because of the limited dynamic range and resolution of the VLB interferometer systems, only the brightest two or three components may be observed. The rather low limits which can be placed on the presence of any third component in 3C 345, or 3C 120 for the 1975 event, do not seem consistent with these multiple component models. Likewise, the consistent position angle which is observed in 3C 120 for several successive outbursts suggests that there is a preferential direction of activity, and that components are not ejected isotropically. The observations of super-light velocities in compact cores which are aligned with extended components would be further evidence against such a model.

Another complication of these models is that they require large kinetic energy in the form of bulk motion, particularly if there are heavy particles as well as electrons traveling at relativistic speeds. Even if 
there is sufficient energy to propel the components with the required relativistic velocities, deceleration by the surrounding medium may be important since the sources are typically smaller than the dense region giving rise to the optical emission lines. This is particularly critical in 3C 120 where the expansion occurs in the dense nucleus of the galaxy. Unless previous events have blasted out a hole, it is difficult to maintain the required high velocities. There is some evidence for rapid deceleration in the expansion of 3C 345 during 1976, but it is surprisingly sudden, and the apparent acceleration which occurred during 1971-1975 is difficult to understand with models of this type.

The simplest and most obvious interpretation of the apparent fasterthan-light motion is that the sources are much closer than indicated by their redshifts and currently accepted cosmological models and constants. This may be considered as evidence of the non-cosmological origin of quasar redshifts. However, the observations of 3C 120 are not readily explained in this way, since $3 C 120$ is identified with an object which appears to be a spiral galaxy (e.g. Arp 1975) whose distance is generally accepted. Since no stellar absorption lines are seen in $3 C 120$, however, its true nature may perhaps be questioned. Indeed if $3 C 120$ is a galaxy at a distance corresponding to its redshift, then the outlying features photographed by Arp would make it one of the largest spiral galaxies known.

\section{Conclusions}

Although some of the earlier VLBI data permitted interpretations of the apparent super-light motion with multi-component stationary models, the more detailed data now available, at least for 3C 120 and 3C 345, appear to require an actual change in component separation by up to a factor of ten. This corresponds to apparent linear velocities of up to 10 times the speed of light if these sources are located at the distance corresponding to their redshift. Thus, these observations provide some evidence against the cosmological distance of these objects. Other, more conventional, interpretations have been suggested, but they are not completely satisfactory in explaining the growing wealth of observational data.

\section{Acknowledgement}

We wish to thank our many colleagues at Caltech and the MPIfR who have collaborated in the observations reported in this paper. 


\section{References}

Arp, H. 1975, Pub. A.S.P., 87, 545.

Cohen, M. H. et al. 1971, Ap. J., 170, 207.

Cohen, M. H. et a1. 1976, Ap. J. (Letters), 206, L1.

Dent, W. A. 1971, private communcation.

Dent, W. A. 1972, Science, 175, 1105.

Dent, W. A. and Kojoian, G. 1972, A. J., 77, 819.

Dent, W. A. et al. 1974, A. J., 79, 1232.

Epstein, R. I. and Geller, M. J. 1976, Nature, in press.

Gubbay, J. et a1. 1969, Nature, 224, 1094.

Hunstead, R. W. 1972, Ap. Letters, 12, 193.

Jones, T. W. and Burbidge, G. R. 1973, Ap. J., 186, 791.

Kellermann, K. I. and Pauliny-Toth, I. I. K. 1968, Ann. Rev. Astr. and Ap., 6, 417 .

Kellermann, K. I. and Pauliny-Toth, I. I. K. 1969, Ap. J. (Letters), 155, L71.

Kellermann, K. I. et a1. 1973, Ap. J. (Letters), 183, L51.

Kellermann, K. I. et al. 1974, Ap. J. (Letters), 189, L19.

Kellermann, K. I. et al. 1977, Ap. J., in press.

Knight, C. A. et a1. 1971, Science, 172, 52.

Legg, T. et al. 1976, Ap. J., in press.

Moffet, A. et a1. 1972, in IAU Symposium No. 44, ed. D. S. Evans, p. 228.

Ozernoi, L. M. and Sazonov, V. N. 1969, Ap. and Space Sci., 3, 395.

Pauliny-Toth, I. I. K. and Kellermann, K. I. 1966, Ap. J., 146, 634.

Pauliny-Toth, I. I. K. et al. 1976, Nature, 259, 17.

Pauliny-Toth, I. I. K. et al. 1977, in preparation.

Rees, M. J. 1967, M.N.R.A.S., 135, 345.

Schilizzi, R. T. et al. 1975, Ap. J., 201, 263.

Seielstad, G. A. 1974, Ap. J., 193, 55.

Shaffer, D. B. 1973, Ph.D. Thesis.

Shaffer, D. B. et a1. 1972, Ap. J. (Letters), 173, L147.

Shaffer, D. B. et al. 1975, Ap. J., 201, 256.

Shaffer, D. B. et al. 1977, in preparation.

Shapiro, I. I. et a1. 1973, Ap. J. (Letters), 183, L47.

Whitney, A. R. et a1. 1971, Science, 173, 225.

Wittels, J. J. et a1. 1976, Ap. J. (Letters), 206, L75. 


\section{DISCUSSION}

J.-C. PECKER: I do not believe, indeed, the "illusion" interpretation; and I am in favour of a non-cosmological distance. Let us assume that radio-astronomers had measured transverse velocities, before optical astronomers had discovered radial velocities; I presume then that the latter would have spoken more easily about "apparent" velocities the former would have spoken about "real velocities". Now, it is the reverse. To keep this historical bias, an enormous ingenuity has been shown by Rees and others - Ingenious indeed, but not necessarily true! K.I. KELLERMANN: I agree! The division of astronomers into heretics and conservatives often depends on who come first.

E.M. BURBIDGE: To add to Dr. Pecker's comment: had the QSOs been discovered before galaxies, and their redshifts plotted against apparent magnitude in a "Hubble diagram" one would never have deduced an expansion of the Universe!

H.E. SMITH: I would like to comment on $3 \mathrm{C} 120$ as a normal galaxy. Margaret Burbidge, Jack Baldwin, Joe Wampler and I have obtained observations of the "disk" of 3C 120. The evidence for a population of late type stars is ambiguous. However, we can say that if present the light must be diluted by the light of young stars or nonthermal radiation. The disk does show line emission, however, the line intensity ratios do not permit this emission to be attributed to normal HII regions but rather are characteristic of nonthermally ionized gas. In fact some of the knots that show up so beautifully on Arp's photographs show only continuous emission. If $3 \mathrm{C} 120$ is a galaxy it is not a normal spiral.

V. PETROSIAN: Do you see any super-light motions not accompanied with flux changes? Suppose the source expands or ejects material into regions where there is already material - then you get radiation from a front as the moving material hits the material in place, and you measure the phase velocity of the front rather than the velocity of motion.

The photograph of $3 \mathrm{C} 120$ shows an object more like the Crab nebula than a spiral galaxy.

K.I. KELLERMANN: Essentially all compact sources which have been monitored 
for any length of time show intensity variations. Structures have been observed for only 10 of these and roughly half show apparent superluminal motion.

P. MORRISON: What star complement would be required to supply measurable $\mathrm{H}$ and $\mathrm{K}$ absorption lines, for example, in $3 \mathrm{C} 120$ ?

G. BURBIDGE: Probably the light from stars must be $30 \%$ or greater if stellar absorptions features are to be seen. Thus, it could be that in all cases of this type a normal galaxy underlies the features which we see. However, there may equally well be no galaxy present. If you assume that there is the burden of proof is on you. The large extension of $3 C 120$ and the abnormal emission lines obvious in the outer parts argue against its being a normal galaxy.

V. RUBIN: A sample of normal Sc galaxies shows diameters of $20-80 \mathrm{kpc}$ $\left(\mathrm{H}=50 \mathrm{~km} \mathrm{~s}^{-1} \mathrm{Mpc}^{-1}\right)$. Therefore it is not unlikely that a spiral at very large distance would lie at the extreme upper limit of the diameter distribution. A diameter of $120 \mathrm{kpc}$ for 3C 120 does not seem abnormal. K.I. KELLERMANN: 3C 120 does not have a very large redshift $(z=0.03)$ and $120 \mathrm{kpc}$ lies well beyond the range of 20-80 kpc.

M. BURBIDGE: Has $21-\mathrm{cm}$ radiation been looked for in $3 \mathrm{C} 120$ ?

M.S. ROBERTS: It has been looked for but not found (to rather poor limits).

J.P. VIGIER: 1) The second interpretation (real physical superlight velocities) is out (in my opinion) at least up to $\mathrm{z} \sim 0.4$ by the results presented in Grenoble by Dr. Baum and his coworkers.

2) There is an evident difficulty in the interpretation as real head-on expansion i.e. the existence of accelerations. How could such a real separation accelerate in Martin Rees' model?

K. I KELLERMANN: I think I said that it was not clear how to explain the apparent accelerations. But I suppose it can be done within the general class of models where you see a signal hitting a curved "screen".

J.M: BARNOTHY: Zwicky has already noted that galaxies are powerful gravitational lenses. If the axis of such a lens passes close to a 
compact radio source, for instance, a supernova shell, it produces two crescent shaped images. As the supernova shell expands, the length of the crescents increases. Seen with a long base line radio interferometer, the crescent will simulate a double radio source with components receeding from each other. (B.A.A.S. 1971, 3 , 472). This explanation can account for the overlight separation velocity of the radio components. as well as why only expanding, but never contracting components were observed. When spiral galaxies in strongly inclined positions act as lenses, they can produce several images from the same source. The scanning motion of the optical axis can produce strong brightness variations of these images while the axis approaches or recedes from the compact radio source (Astron. J. 11, 155, 1966). 